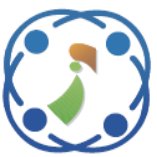

\title{
Detection and Classification of Indonesian Civet and Non-Civet Coffee Based on Statistical Analysis Comparison Using E-Nose
}

\author{
Sulaiman Wakhid ${ }^{1}$ \\ Riyanarto Sarno ${ }^{1,2 *}$ \\ Shoffi Izza Sabilla ${ }^{2}$ \\ Dike Bayu Maghfira ${ }^{1}$ \\ ${ }^{I}$ Department of Technology Management, Faculty of Creative Design and Digital Business, \\ Institut Teknologi Sepuluh Nopember (ITS) Sukolilo, Surabaya 60111, Indonesia \\ ${ }^{2}$ Informatics Department, Faculty of Intelligent Electrical and Informatics Technology, \\ Institut Teknologi Sepuluh Nopember (ITS) Sukolilo, Surabaya 60111, Indonesia \\ * Corresponding author's Email: riyanarto@if.its.ac.id
}

\begin{abstract}
Civet coffee is a highly priced premium beverage in Indonesia. Because of its high economic value, civet coffee is often falsified with non-civet coffee. The detection and classification of coffee aroma using an e-nose has been the subject of several researches. However, only few researches have been done on civet coffee and non-civet coffee detection using an e-nose. This study aimed to improve the classification between civet coffee and non-civet coffee by trying out different combinations of classification methods and statistical parameters. The coffee aroma data were taken from e-nose sensors with different sensitivity toward certain chemicals. There are a number of steps in the classification of coffee aroma: ground truth data acquisition, statistical feature extraction, classification, and performance evaluation. The experimental results of this study indicate that an e-nose can recognize and distinguish well between civet and non-civet coffee. Comparing 6 classes of coffee, the best performing combination was the decision tree algorithm with the average and standard deviation parameters, which obtained $97 \%$ accuracy.
\end{abstract}

Keywords: Civet coffee, E-nose, Sensor, Classification, Machine learning.

\section{Introduction}

Coffee is one of the most traded plantation commodities in the world. As a tropical country, Indonesia produces a large number of coffee varieties, of which Arabica and Robusta are the main types. Apart from these two types, there is also a premium coffee that is known for its outstanding quality, namely civet coffee. This coffee is notoriously expensive because civet coffee beans are biologically processed in the digestion tract of Asian palm civets (Paradoxurus Hermaphroditus) [1]. Civets instinctively choose the ripest and best coffee cherries to eat. A fermentation process occurs in their digestive tract, which chemically modifies the cherries and gives the coffee a unique taste. This long and rare process results in the price of Civet coffee in the market being about 2-3 times higher than the price of other premium coffees [2]. Its high demand and very limited supply provide an opportunity for fraudulent coffee traders to falsify civet coffee with non-civet coffee. This is illegal and harms consumers.

All types of coffee have a specific aroma so that in addition to their taste they can also be distinguished through their aroma [3]. Distinguishing civet coffee from non-civet coffee is not easy for nonexperts. Civet coffee and non-civet coffee have different authentic scents. In principle, the difference in aroma of civet coffee and non-civet coffee makes it possible to distinguish between falsified civet coffee and real civet coffee $[4,5]$. E-noses use a sensor array to obtain aroma signal data, from which statistical parameter values can be extracted for classification [6,7]. Several previous studies have shown the ability of e-noses to distinguish between Arabica and Robusta coffee with an accuracy of $71 \%$ [8]. Another research has shown that Philippine civet and non-civet coffee could be distinguished by an enose using principal component analysis (PCA) [9]. 
Hence, an e-nose (electronic nose) is expected to be able to detect falsification of civet coffee.

The purpose of the present research was to improve the classification between civet coffee and non-civet coffee using an e-nose device. Civet and non-civet coffee data from 3 different regions in Indonesia, i.e. Aceh, Arjuno Malang, and Bengkulu, were used. Thus, there was a total of 6 classes, i.e. Aceh civet coffee (LA), Aceh non-civet coffee (NLA), Arjuno civet coffee (LAR), Arjuno non-civet coffee (NLAR), Bengkulu civet coffee (LB) and Bengkulu non-civet coffee (NLB). The use of 6 classes aimed to improve upon a previous research on the classification of coffee using an e-nose, which used only 2 classes [8].

A comparative analysis was conducted between several classification methods using different statistical parameters to find the best combination for the classification of civet and non-civet coffee. The following statistical parameters were used: average, standard deviation, minimum and maximum value. The values of the statistical parameters were obtained from the stationary signal data generated by the enose device from the aroma of coffee samples. The statistical parameters were classified using four different supervised learning methods, i.e. logistic regression, support vector machine (SVM), decision tree, and naïve Bayes. The classification results were evaluated using k-fold validation [10] along with a confusion matrix to obtain precision, accuracy, and f1 score.

This paper is organized into the following sections: Section 1 and 2 explain the background of the research topic and previous works related to it. Section 3 gives a detailed description of the methods used in the experiment. Section 4 describes the results of the experiment. Section 5 evaluates the results and performance of the methods. Finally, the last section provides the conclusion of this work.

\section{Related literature}

Several previous studies related to the classification of coffee aroma using an e-nose have been conducted with various objectives and using various methods. One research compared the sensory assessment of a panel and an e-nose in recognizing and measuring the aroma of different varieties of coffee [11]. This study used Temporal Dominance of Sensations (TDS) for 15 sensory panel techniques. The data received by the e-nose were reduced and converted to 2-dimensional form using principal component analysis. The results showed similarities between the assessments made by humans and by the e-nose device in recognizing seven coffee varieties.
The aromas of Robusta and Arabica coffee have previously been classified using an e-nose [8]. In this study, an Arduino development board was used along with an MQ 135 gas sensor circuit to detect the aroma of ground coffee. The aroma of coffee was converted by the Arduino to a digital signal. Classification of the aromas of Robusta and Arabica was done using support vector machine (SVM) and the Perceptron method. Evaluation of the research results showed that the SVM method had $71 \%$ accuracy while Perceptron had $57 \%$ accuracy.

Monitoring of the coffee roasting process has been done in another study [12] using an e-nose device with a gas sensor array that was integrated in a mini-batch coffee roaster. This device captured data on the humidity, the temperature, and the aroma of the coffee. The experiment showed that the gas sensor could capture the coffee aroma pattern, which was then analyzed using PCA. However, the PCA scatter results did not produce a discernible pattern so that the distinction between light, medium, and dark (LMD) as a result of coffee roasting could not be made.

Another study classified 7 Chinese Robusta coffee cultivars prepared with different levels of roasting [13]. This study used an e-nose and an etongue to obtain the characteristics of each variety. Coffee data were taken from 126 samples of roasted coffee beans distributed in Hainan province, China. The classification methods used were PCA, Knearest neighbor analysis (KNN), partial least squares discriminant analysis (PLS-DA), and artificial neural back-propagation (BP-ANN). The analysis showed that the results of BP-ANN using PLSR regression were better than those from the other methods, with a $94.0 \%$ recognition rate.

Research on the aroma of civet coffee has been carried out using an e-nose combined with gas chromatography mass spectrometry (GCMS). The research used 4 brands of civet coffee, both of the Arabica and the Robusta variety, originating from the Philippines. The sensor configuration used was a series of metal oxide (MOX) sensors. Using PCA and cluster analysis a grouping was made between civet and non-civet coffee. The clusters of civet and noncivet coffee samples confirmed that the characteristic aroma is an important indicator in recognizing coffee varieties [9].

In addition to the comparison of classification methods, the present study also focused on a comparison of the effects of using different statistical parameters. Some other studies that used e-noses utilized statistical parameters to classify the signal data, for example, a research on the detection of diabetes through the analysis of gases produced by 
human breath [14]. A study on the use of an e-nose to predict methane gas on chili utilized the following statistical parameters: average, standard deviation, kurtosis, and skewness [15]. The statistical parameters used in this study were: average, standard deviation, minimum, and maximum value. The statistical parameters were obtained from the response over time of a stationary gas signal.

\section{Proposed methods}

This study aimed to analyze the classification performance of civet and non-civet coffee aroma using an e-nose device. To get the best classification accuracy, a comparison was made between the performance of different combinations of classification methods and statistical parameters. A scheme of the classification system used in this study is shown in Fig. 1.

Raw data were obtained from the sensor array in the e-nose device. The raw data were in the form of digital signals, which were extracted to obtain the statistical parameter values. To get predictive results, the data were entered into several classification algorithms. The classification results were evaluated to determine the accuracy and performance of the methods and parameters used. This process was repeated using different combinations of classification methods and statistical parameters to get the best performing combination.

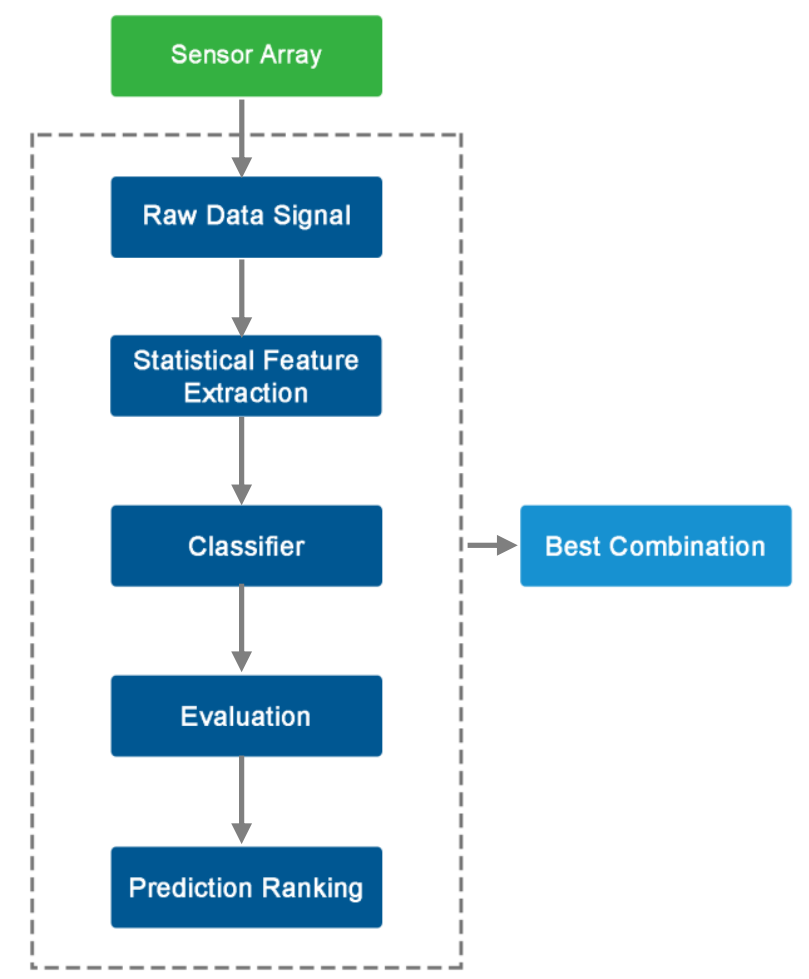

Figure. 1 System scheme to get the best classification method

\subsection{Statistics feature extraction}

The concentration of gases contained in the aroma of coffee is a stationary signal that is captured by the sensor array of the e-nose. From the stationary signal, the statistical parameters can be obtained [14]. In this study, four statistical parameters were used: average, standard deviation, maximum, and minimum value.

To get the average, the signal frequency of each sample is summed and divided by the amount of sample data taken (n), as expressed in Eq. (1).

$$
\operatorname{Average}(\bar{x})=\frac{1}{n}\left(\sum_{i=1}^{n} x_{i}\right)
$$

The get the standard deviation, the distribution of the signal frequency data from the sample is determined. If the value of $\sigma$ is high, then the values of the data spread from the middle value of $\bar{x}$. The standard deviation is given by Eq. (2).

$$
\text { Standard Deviation }(\sigma)=\sqrt{\frac{\sum_{i=1}^{n}(x i-\mu)^{2}}{n-1}}
$$

The maximum parameter value is the highest value of the data distribution while the minimum parameter value is the lowest value, as expressed in Eq. (3) and Eq. (4).

$$
\begin{aligned}
& \text { Maximum }=\max \left(x_{i}\right) \\
& \text { Minimum }=\min \left(x_{i}\right)
\end{aligned}
$$

The statistical parameter values were calculated for each sensor, as shown in Fig. 2. Because 4 gas sensors were used, 16 statistical parameters were obtained.

\subsection{Classification method}

This study conducted a comparison of four methods to classify between civet and non-civet coffee, i.e. logistic regression, support vector machine, decision tree classifier (DTC), and naïve Bayes. Logistic regression is a method that classifies data items into categories, where the target is a binary number [16]. This binary number contains data that are classified as 1 or 0 , yes or no, true or false. In the case of this study, the binary number refers to coffee aroma classified as civet coffee or non-civet coffee.

\begin{tabular}{|c|c|c|c|c|c|c|c|}
\hline I & Avg & Std & Max & Min & & Avg & Std \\
D & MQ2 & MQ2 & MQ2 & MQ2 & $\cdots$ & MQ4 & MQ4 \\
\hline
\end{tabular}

Figure. 2 Statistical parameter attributes 


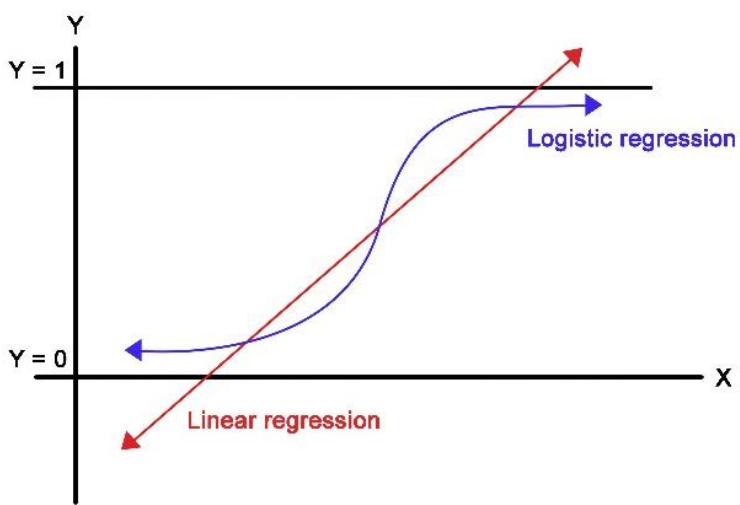

Figure. 3 Linear and logistic regression curve

Simple logistic regression is a nonlinear transformation of linear regression that has an Sshaped distribution function, as shown in Fig. 3. The formula of a logistic regression can be as follows in Eq. (5).

$$
\sigma(x)=\frac{1}{1+\exp ^{-(x)}}
$$

The purpose of the logistic regression in this study was to find the best model to describe the relationship between dichotomous characteristics (dependent variable) and a set of independent variables (predictor or explanatory).

Support vector machine is a supervised learning algorithm that analyzes data and recognizes patterns, which can be used for classification and regression analysis [17-19]. SVM was first designed for binary classification. Given a training set $T=\left\{\left(x_{i}, y_{i}\right)_{N}\right\}$, where $y_{i} \in\{+1,-1\}$, the goal of binary classification is to identify a hyperplane, an affine subspace of dimension $N-1$, such that this hyperplane can divide the space into two halves with respect to the inputs of two distinct classes. In this case the hyperplane divides the civet and non-civet classes. The best hyperplane is located in between the two data sets that represent the two classes, as shown in Fig. 4.

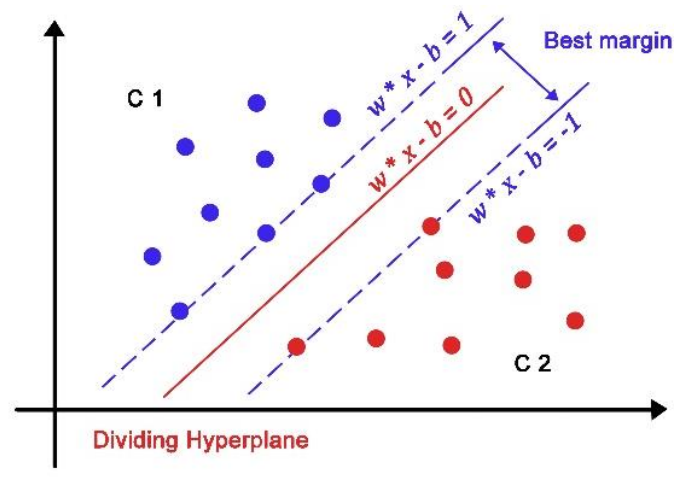

Figure. 4 SVM dataset representation and margin
The margin between the two classes can be calculated by finding the distance between the two supporting hyperplanes of both classes [20]. The margin is calculated by Eq. (6).

$$
\left(\frac{w}{\|w\|}\left(x_{1}-x_{2}\right)\right)=\frac{2}{\|w\|}
$$

Decision tree classifier (DTC) is another machine learning technique, which works with recursive partitioning of datasets to achieve a homogeneous classification of the target variables. This algorithm works at the moment of each separation to reduce the entropy of the target variable in the dataset. The entropy can be obtained by Eq. (7).

$$
E(S)=\sum_{i=1}^{n}-p_{i} \log _{2} p_{i}
$$

Hence, it can be generated by choosing the optimal separation of several independent variables. The main advantage of this method is that it is not computationally expensive, does not make assumptions about the distribution of environmental and strong variables for missing data and excessive environmental variables [21, 22].

The naïve Bayes classifier is a simple classification method developed by Thomas Bayes to predict future probabilities based on past experience. It works by calculating a set of probabilities by adding up the frequency and combination of values from a given dataset. The Bayes theorem assumes that all attributes are independent, or not interdependent, given by value in class variables. Simply put, if there are two separate events, in this case $x$ and $h$, then the Bayes theorem is as formulated as Eq. (8).

$$
P\left(h_{j} \mid x\right)=\frac{p\left(x \mid h_{j}\right)}{p(x)} p\left(h_{j}\right)
$$

This classifier consolidates the presence or absence of certain features that are not relevant to the current situation. Naïve Bayes is a supervised learning algorithm, so classification requires training data to be able to predict and detect attributes [23, 24].

\section{Results and discussion}

\subsection{Data acquisition}

The e-nose device used in this study was designed using an Arduino Mega 256 board with MQ series sensors. The testing room was a container with air circulation to break down the aroma of the coffee. 


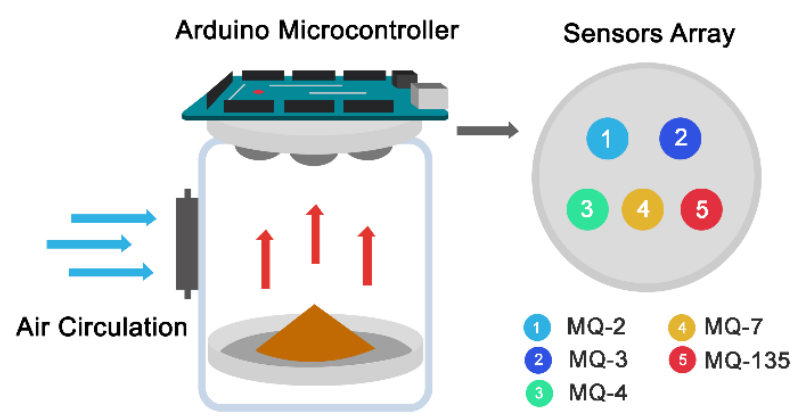

Figure. 5 E-Nose system design for data acquisition

Table 1. Sensors and measured gas contents used in the enose system

\begin{tabular}{ll}
\hline \multicolumn{1}{c}{ Sensor } & \multicolumn{1}{c}{ Measured Gas Contents } \\
\hline MQ-135 & Carbon dioxide \\
MQ-2 & $\begin{array}{l}\text { Methane, propane, LPG, i-butane, } \\
\text { alcohol, H2, smoke }\end{array}$ \\
MQ-3 & CO, benzene, alcohol, methane, \\
hexane, LPG \\
MQ-4 & Natural gas, methane \\
MQ-7 & CO \\
\hline
\end{tabular}

The gas was captured by the sensor array to be processed by the Arduino into a digital signal. The results of the digital signal produced 5 outputs originating from each sensor, as shown in Fig. 5. Each sensor had different sensitivity specifications [25] as shown in Table 1.

This study used two types of coffee, i.e. civet Arabica coffee and original Arabica coffee. Each type of coffee was taken from three different regions in Indonesia, namely Aceh, Arjuno Malang, and Bengkulu. The coffee used was ground coffee with an ideal grinding level from rough to medium. Data were collected 50 times for each type of coffee. Temperature data collection was carried out at room temperature (between $20^{\circ} \mathrm{C}$ and $25^{\circ} \mathrm{C}$ ). Each take was carried out for 15 minutes with a weight of 15 grams of coffee.

The coffee samples consisted of two types, originating from three different regions, The following categorization was made to facilitate classification: the Aceh Arabica Coffee was divided into 2 class categories, called Aceh civet coffee (LA) and Aceh non-civet coffee (NLA); the Arjuno Malang Arabica coffee was divided into 2 class categories, called Arjuno civet coffee (LAR) and Arjuno non-civet coffee (NLAR); the Bengkulu Arabica coffee was divided into 2 class categories, called Bengkulu civet coffee (LB) and Bengkulu noncivet coffee (NLB). Hence, a total of 6 classes was used in this study.

Aroma detection was done using an e-nose device by placing 15 grams of ground coffee into the sensor

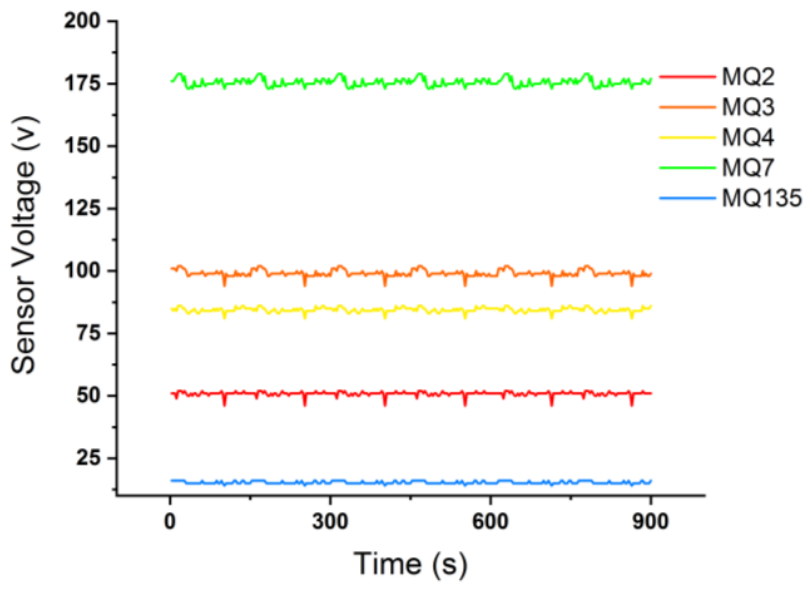

Figure. 6 Result of raw signal LA

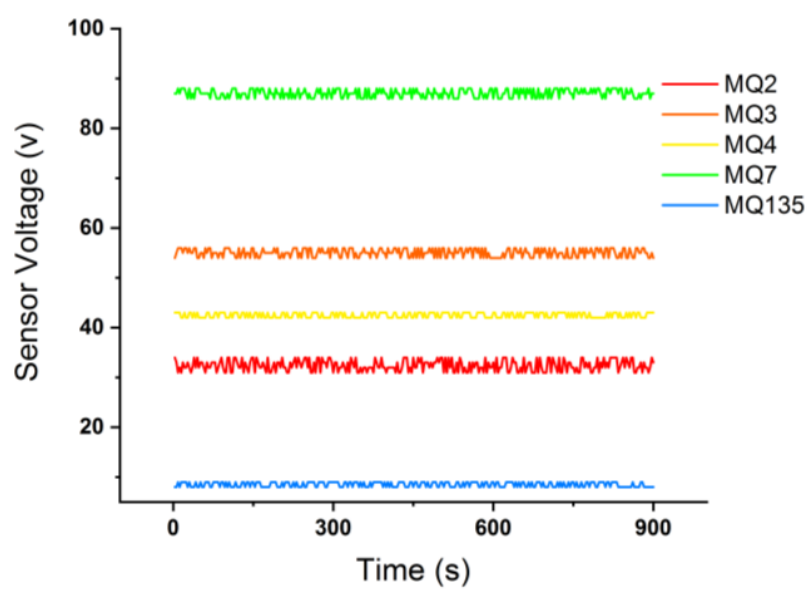

Figure. 7 Result of raw signal NLA

chamber of the e-nose device. The coffee aroma that came out was the input for the sensors (MQ-135, MQ-2, MQ-3, MQ-4, and MQ-7) which transmitted their signals to the Arduino microcontroller. The microcontroller converted the analog input signals to digital signals. Fig. 6 and Fig. 7 illustrate the frequency of the raw data signals from the LA and NLA classes. Each class had a set of 50 data so that there was a total of 600 samples from all classes used in this study.

\subsection{Feature extraction of statistical parameters}

The sampling results from the six coffee categories produced data in the form of frequency distributions. The signal distribution was normalized to the level of the signal value. Averaging was done by using the following statistical parameters: average, standard deviation, minimum, and maximum. Calculations were performed on each column referring to the sensor name on the e-nose device, as shown in Table 2. 
Table 2. Extraction results of statistical parameters from each sensor in the LA and NLA classes

\begin{tabular}{|c|c|c|c|c|c|}
\hline \multirow[b]{2}{*}{ No. } & \multirow[b]{2}{*}{ Class } & \multicolumn{4}{|c|}{ Sensors } \\
\hline & & $\begin{array}{r}\text { Avg } \\
\text { MQ2 } \\
\end{array}$ & $\begin{array}{c}\text { Std } \\
\text { MQ2 } \\
\end{array}$ & $\begin{array}{c}\text { Min } \\
\text { MQ2 } \\
\end{array}$ & $\begin{array}{l}\text { Max } \\
\text { MQ2 } \\
\end{array}$ \\
\hline 1 & LA & 50.90 & 0.92 & 46 & 52 \\
\hline 2 & LA & 54.72 & 1.99 & 51 & 57 \\
\hline$\ldots$ & $\ldots$ & $\ldots$ & $\ldots$ & $\ldots$ & $\ldots$ \\
\hline 50 & LA & 51.20 & 1.30 & 49 & 53 \\
\hline 51 & NLA & 32.41 & 1.11 & 31 & 34 \\
\hline$\ldots$ & $\ldots$ & $\ldots$ & $\ldots$ & $\ldots$ & $\ldots$ \\
\hline 100 & NLA & 32.49 & 0.50 & 32 & 33 \\
\hline
\end{tabular}

Table 3. Classifier performance result in scenario with 2 classes

\begin{tabular}{cccc}
\hline \multirow{2}{*}{ Method } & \multicolumn{3}{c}{ Accuracy } \\
\cline { 2 - 4 } & LA-NLA & LAR-NLAR & LB-NLB \\
\hline Logistic & 0.95 & 1.00 & 1.00 \\
Regression & 1.00 & 1.00 & 1.00 \\
SVM & 1.00 & 1.00 & 1.00 \\
Naïve & & 1.00 & 1.00 \\
Bayes & 0.95 & & \\
Decision & Tree & &
\end{tabular}

Table 4. Classifier performance result in scenario with 6 classes

\begin{tabular}{cc}
\hline Method & Accuracy \\
\hline Logistic Regression & 0.95 \\
SVM & 0.87 \\
Naïve Bayes & 0.90 \\
Decision Tree & 0.90 \\
\hline
\end{tabular}

Each sample produced one line of statistical parameter data. Thus, each class had 50 statistical parameters data taken from 50 experiments for each class.

\subsection{Classification result}

Before entering the classification phase, the data were divided into two parts using cross-validation. The data were divided into training data $(80 \%)$ and testing data (20\%). Data testing was carried out crosssectionally using a k-fold value of 10 [26]. The methods used for classification were logistic regression, support vector machine (SVM), decision tree classifier (DTC), and naïve Bayes classifier. There were two experimental scenarios, namely one scenario using 2 classes and one scenario using 6 classes.

The experiment using 2 classes was carried out by comparing two types of coffee, namely civet and noncivet Arabica coffee originating from the same region.
The experiment using 6 classes was carried out by combining all classes into one for classification.

The classification results were measured based on the accuracy obtained by each method. The accuracy value illustrates how accurately the system classified correctly. The accuracy value was obtained from a comparison between the correct classification results and the overall data. Eq. (9) is the method used to get the level of accuracy in this study, distinguishing between true positive (TP), true negative (TN), false positive (FP), and false negative (FN). These are the provisions from the classification assessment by the confusion matrix.

$$
\text { Accuracy }=\frac{(T P+T N)}{(T P+T N+F P+F N)} \times 100 \%
$$

Based on the 2-class experiment, the highest accuracy level was $100 \%$ while the lowest was $95 \%$, as shown in Table 3. These results indicate that Arabica civet coffee and non-civet coffee could be classified properly by the system. In the 6-class experiment, the accuracy results ranged from $85 \%$ to $95 \%$, as shown in Table 4 . Thus, the accuracy in the 6-class experiment was lower compared to the 2-class experiment. This proves that the use of more classes increases the level of difficulty for the classification system.

\section{Evaluation of results and performance}

Confusion matrix is a method commonly used for evaluating the results of machine learning classifications that have more than one class [27]. In this study, a confusion matrix was used to calculate the level of accuracy of the methods used. A confusion matrix contains information related to actual class data and predicted class data.

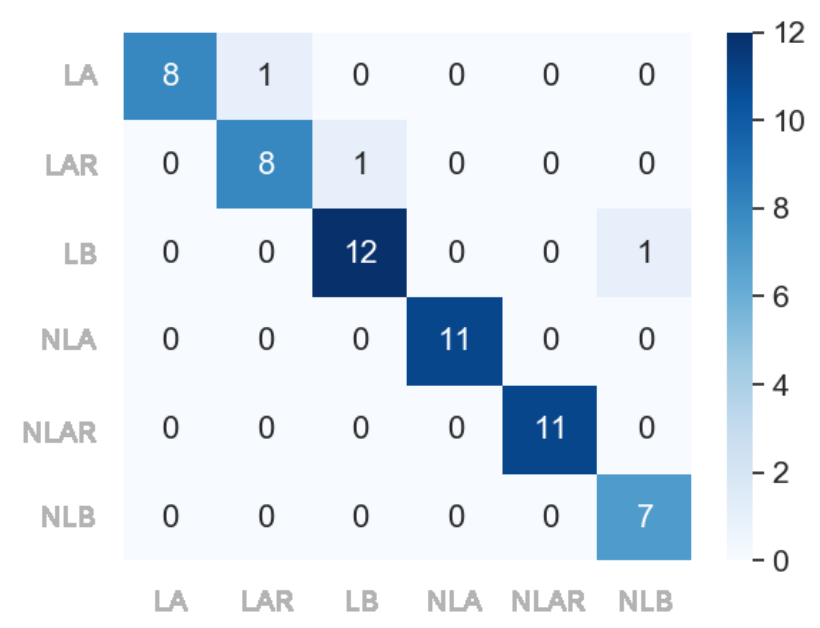

Figure. 8 Confusion matrix of 6-class scenario 


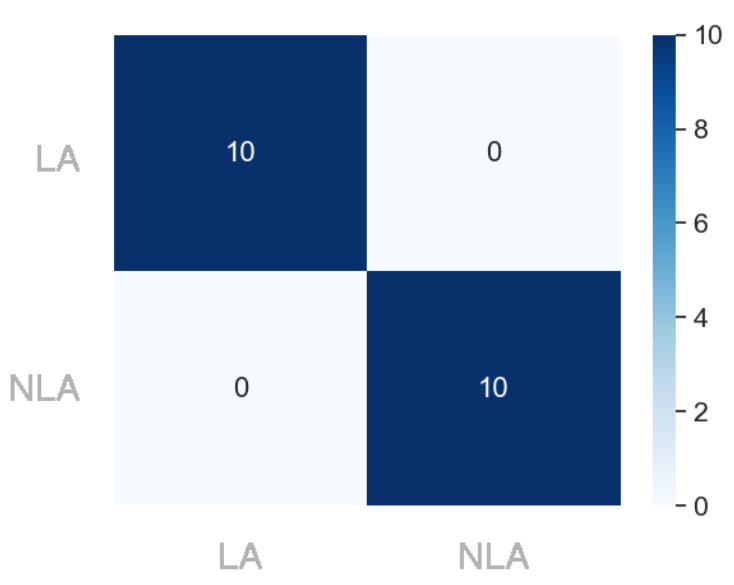

Figure. 9 Confusion matrix of 2-class scenario

Fig. 8 and Fig. 9 show the confusion matrix from the scenarios using 6 classes and 2 classes. The results of the 6-class scenario show that non-civet coffee, indicated by NLA, NLAR, NLB, had perfect classification results. On the other hand, civet coffee had some prediction errors. The LA class had one data that was misclassified as LAR, while the LAR class had one data misclassified as LB class. The LB class had one data that was included in the NLB class. However, the experiment using the 2-class scenario had a perfect score, where 10 data were classified as LA class and the rest as NLA class.

Based on the confusion matrix provisions, performance indicator values can be obtained in the form of accuracy, precision, and recall [28]. The precision value reflects the number of data categorized as positive that are correctly classified divided by the total number of data classified as positive, as expressed in Eq. (10). The recall value describes the percentage of the category of positive data that are correctly classified, as expressed in Eq. (11).

$$
\begin{gathered}
\text { Precision }=\frac{T P}{(T P+F P)} \times 100 \% \\
\text { Recall }=\frac{T P}{(T P+F N)} \times 100 \%
\end{gathered}
$$

From one of the sample calculations in Table 5, where 6 classes were used, the true positive rate (TPR) was $96 \%$, the false positive rate (FPR) was $1 \%$, the false negative rate (FNR) was $4 \%$, and the true negative rate (TNR) was $99 \%$. By evaluating the results of each classification method, the performance of the e-nose system could be improved [29].
Table 5. Performance measurement of Decision Tree method in 6-class scenario

\begin{tabular}{ccccccc}
\hline & \multicolumn{7}{c}{ Class } \\
\cline { 2 - 7 } & LA & LAR & LB & NLA & NLAR & NLB \\
\hline Support & 9 & 9 & 13 & 11 & 11 & 7 \\
TP & 8 & 8 & 13 & 11 & 11 & 7 \\
FP & 0 & 1 & 1 & 0 & 0 & 0 \\
FN & 1 & 1 & 0 & 0 & 0 & 0 \\
TN & 51 & 50 & 46 & 49 & 49 & 53 \\
Precision & 1.00 & 0.89 & 0.93 & 1.00 & 1.00 & 1.00 \\
Recall & 0.89 & 0.89 & 1.00 & 1.00 & 1.00 & 1.00 \\
F-1 & 0.94 & 0.89 & 0.96 & 1.00 & 1.00 & 1.00 \\
Score & & & & & & \\
TPR & 0.96 & & & & & \\
FPR & 0.01 & & & & & \\
FNR & 0.04 & & & & & \\
TNR & 0.99 & & &
\end{tabular}

This study also performed a comparison between the classification methods with different statistical parameters. This comparison was used to obtain the best outcome among the four methods for different parameter combinations. The first combination was average and standard deviation (Avg-Std). The second combination was minimum and maximum value (Min-Max). The third combination was all statistical parameters, i.e. average, standard deviation, minimum, and maximum (Avg-Std-Min-Max). For each combination the accuracy value was calculated with each of the 4 classification methods.

\begin{tabular}{|c|c|c|c|c|}
\hline \multirow[b]{2}{*}{ Method } & \multirow{2}{*}{$\begin{array}{c}\text { Statistic } \\
\text { Parameter }\end{array}$} & \multicolumn{3}{|c|}{ Accuracy } \\
\hline & & $\begin{array}{l}\text { LA- } \\
\text { NLA }\end{array}$ & $\begin{array}{l}\text { LAR- } \\
\text { NLAR } \\
\end{array}$ & $\begin{array}{l}\text { LB- } \\
\text { NLB } \\
\end{array}$ \\
\hline \multirow{3}{*}{$\begin{array}{c}\text { Logistic } \\
\text { Regression }\end{array}$} & Avg-Std & 1.00 & 1.00 & 1.00 \\
\hline & Min-Max & 0.95 & 1.00 & 1.00 \\
\hline & $\begin{array}{l}\text { Avg-Std- } \\
\text { Min-Max }\end{array}$ & 0.95 & 1.00 & 1.00 \\
\hline \multirow{3}{*}{ SVM } & Avg-Std & 1.00 & 1.00 & 1.00 \\
\hline & Min-Max & 1.00 & 1.00 & 1.00 \\
\hline & $\begin{array}{l}\text { Avg-Std- } \\
\text { Min-Max }\end{array}$ & 1.00 & 1.00 & 1.00 \\
\hline \multirow{3}{*}{$\begin{array}{l}\text { Naïve } \\
\text { Bayes }\end{array}$} & Avg-Std & 1.00 & 1.00 & 1.00 \\
\hline & Min-Max & 1.00 & 1.00 & 1.00 \\
\hline & $\begin{array}{l}\text { Avg-Std- } \\
\text { Min-Max }\end{array}$ & 1.00 & 1.00 & 1.00 \\
\hline \multirow{3}{*}{$\begin{array}{l}\text { Decision } \\
\text { Tree }\end{array}$} & Avg-Std & 0.95 & 1.00 & 0.95 \\
\hline & Min-Max & 0.95 & 1.00 & 1.00 \\
\hline & $\begin{array}{l}\text { Avg-Std- } \\
\text { Min-Max }\end{array}$ & 0.95 & 1.00 & 1.00 \\
\hline
\end{tabular}

Table 6. Accuracy comparison in 2-class experiment 
Table 7. Accuracy comparison in 6-class experiment

\begin{tabular}{ccc}
\hline Method & Statistic Parameter & Accuracy \\
\hline \multirow{2}{*}{ Logistic } & Avg-Std & 0.92 \\
Regression & Min-Max & 0.88 \\
& Avg-Std-Min-Max & 0.95 \\
& Avg-Std & 0.88 \\
SVM & Min-Max & 0.87 \\
& Avg-Std-Min-Max & 0.87 \\
Naïve & Avg-Std & 0.90 \\
Bayes & Min-Max & 0.88 \\
& Avg-Std-Min-Max & 0.90 \\
Decision & Avg-Std & 0.97 \\
Tree & Min-Max & 0.93 \\
& Avg-Std-Min-Max & 0.90 \\
\hline
\end{tabular}

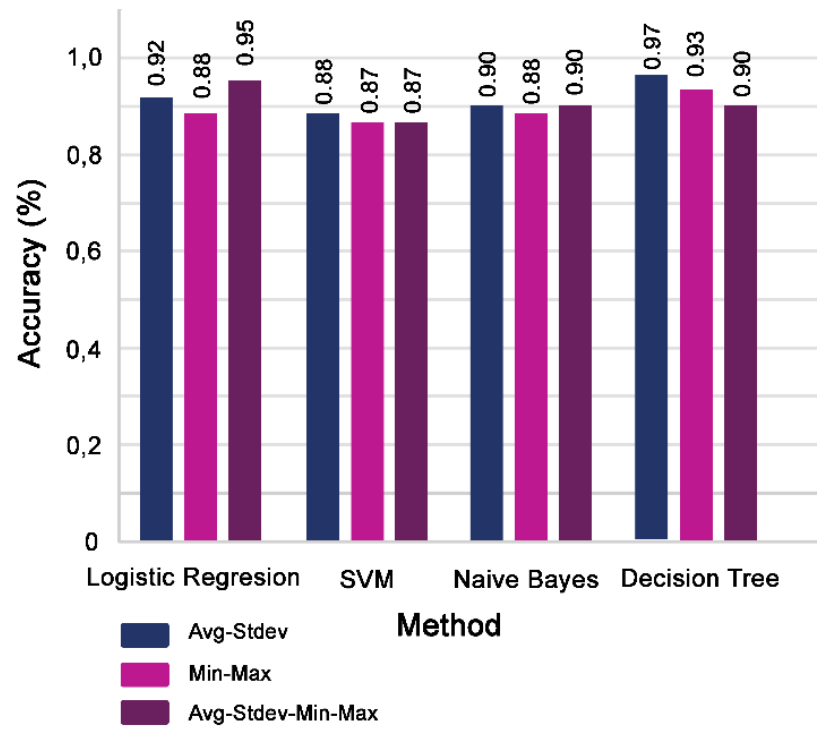

Figure. 10 Comparison chart between statistical methods and parameters

The experiment using comparisons between civet and non-civet coffee from the same origin in Table 6 delivered satisfying results. The LAR and NLAR classes were classified with an accuracy of $100 \%$. For the LB and NLB classes there was one instance that had an accuracy of 95\%. For the LA and NLA classes the best results were shown by classification using the SVM and naïve Bayes methods. In the experiment with 6 classes in Table 7, the highest accuracy level was obtained by the DTC method (97\%), while the lowest accuracy was obtained by the SVM and naïve Bayes methods (87\%).

The use of appropriate statistical parameters affects the accuracy of the classification results. Based on the comparison in Fig. 10, the best average results were produced by using the combinations Avg-Std and Avg-Std-Min-Max, with accuracy values of $97 \%$ and $95 \%$, respectively. These results were better than those from previous studies that also used an Arduino e-nose device with SVM, where the level of accuracy obtained was only $71 \%$ [8].

\section{Conclusion}

The performance of several combinations of classification algorithms and statistical parameters was obtained. The best performance was produced by the combination of the decision tree classification algorithm with the average and standard deviation parameters when comparing 6 classes of coffee (97\%). When comparing 2 classes, the level of accuracy was $100 \%$. The number of comparison classes influences the level of accuracy of the method. For this reason, further research can be done with more classes by mixing civet coffee with non-civet coffee from different regions.

\section{Acknowledgments}

The authors would like to thank Institut Teknologi Sepuluh Nopember for supporting this research.

\section{References}

[1] U. Jumhawan, S. P. Putri, Yusianto, T. Bamba, and E. Fukusaki, "Application of gas chromatography/flame ionization detectorbased metabolite fingerprinting for authentication of Asian palm civet coffee (Kopi Luwak)", Journal of Bioscience and Bioengineering, Vol. 120, No. 5, pp. 555-561, 2015.

[2] U. Jumhawan, S. P. Putri, Yusianto, T. Bamba, and E. Fukusaki, "Quantification of coffee blends for authentication of Asian palm civet coffee (Kopi Luwak) via metabolomics: A proof of concept", Journal of Bioscience and Bioengineering, 2016.

[3] F. Kulapichitr, C. Borompichaichartkul, I. Suppavorasatit, and K. R. Cadwallader, "Impact of drying process on chemical composition and key aroma components of Arabica coffee", Food Chemistry, Vol. 291, pp. 49-58, 2019.

[4] K. Lopetcharat, F. Kulapichitr, I. Suppavorasatit, T. Chodjarusawad, A. Phatthara-Aneksin, S. Pratontep, and C. Borompichaichartkul, "Relationship between overall difference decision and electronic tongue: Discrimination of civet coffee", Journal of Food Engineering, Vol. 180, pp. 60-68, 2016. 
[5] W. Dong, R. Hu, Y. Long, H. Li, Y. Zhang, K. Zhu, and Z. Chu, "Comparative evaluation of the volatile profiles and taste properties of roasted coffee beans as affected by drying method and detected by electronic nose, electronic tongue, and HS-SPME-GC-MS", Food Chemistry, Vol. 272, pp. 723-731, 2019.

[6] D. R. Wijaya, R. Sarno, E. Zulaika, and S. I. Sabila, "Development of mobile electronic nose for beef quality monitoring", In: Procedia Computer Science, Vol. 124, pp. 728-735, 2017.

[7] D. R. Wijaya, R. Sarno, and E. Zulaika, "Sensor Array Optimization for Mobile Electronic Nose: Wavelet Transform and Filter Based Feature Selection Approach", International Review on Computers and Software (IRECOS), Vol. 11, No. 8, pp. 659, 2016.

[8] D. B. Magfira and R. Sarno, "Classification of Arabica and Robusta coffee using electronic nose", In: Proc. of 2018 International Conference on Information and Communications Technology, ICOIACT 2018, Vol. 2018-January, pp. 645-650, 2018.

[9] E. Ongo, M. Falasconi, G. Sberveglieri, A. Antonelli, G. Montevecchi, V. Sberveglieri, I. Concina, and F. Sevilla, "Chemometric discrimination of philippine civet coffee using electronic nose and gas chromatography mass spectrometry", Procedia Engineering, Vol. 47, pp. 977-980, 2012.

[10] T. T. Wong, "Parametric methods for comparing the performance of two classification algorithms evaluated by k-fold cross validation on multiple data sets", Pattern Recognition, Vol. 65, pp. 97107, 2017.

[11] V. Vietoris, P. Zajác, J. Čapla, A. Mendelová, K. Križanová, and L. Benešová, "Comparison of coffee species by sensory panel and electronic nose", Journal of Microbiology, Biotechnology and Food Sciences, Vol. 5, No. 3, pp. 234-237, 2016.

[12] Radi, M. Rivai, and M. H. Purnomo, "Study on electronic-nose-based quality monitoring system for coffee under roasting", Journal of Circuits, Systems and Computers, Vol. 25, No. 10, 2016.

[13] W. Dong, J. Zhao, R. Hu, Y. Dong, and L. Tan, "Differentiation of Chinese robusta coffees according to species, using a combined electronic nose and tongue, with the aid of chemometrics", Food Chemistry, Vol. 229, pp. 743-751, 2017.

[14] Hariyanto, R. Sarno, and D. R. Wijaya, "Detection of diabetes from gas analysis of human breath using e-Nose", In: Proc. of the 11th International Conference on Information and Communication Technology and System, ICTS 2017, Vol. 2018-January, pp. 241-246, 2018.

[15] S. I. Sabilla and R. Sarno, "Development of wavelet transforms to predict methane in chili using the electronic nose", In: Proc. of ICAMIMIA 2017: International Conference on Advanced Mechatronics, Intelligent Manufacture, and Industrial Automation, pp. 271-276, 2018.

[16] C. Zhu, C. U. Idemudia, and W. Feng, "Improved logistic regression model for diabetes prediction by integrating PCA and Kmeans techniques", Informatics in Medicine Unlocked, pp. 100179, 2019.

[17] S. Qiu, J. Wang, C. Tang, and D. Du, "Comparison of ELM, RF, and SVM on E-nose and E-tongue to trace the quality status of mandarin (Citrus unshiu Marc.)", Journal of Food Engineering, Vol. 166, pp. 193-203, 2015.

[18] K. Mahmodi, M. Mostafaei, and E. MirzaeeGhaleh, "Detection and classification of dieselbiodiesel blends by LDA, QDA and SVM approaches using an electronic nose", Fuel, Vol. 258, 2019.

[19] D. R. Wijaya, R. Sarno, and E. Zulaika, "Noise filtering framework for electronic nose signals: An application for beef quality monitoring", Computers and Electronics in Agriculture, Vol. 157, pp. 305-321, 2019.

[20] M. Jupri and R. Sarno, "Taxpayer compliance classification using C4.5, SVM, KNN, Naive Bayes and MLP”, In: Proc. of the 2018 International Conference on Information and Communications Technology, ICOIACT 2018, Vol. 2018-January, pp. 297-303, 2018.

[21] A. Beucher, A. B. Møller, and M. H. Greve, "Artificial neural networks and decision tree classification for predicting soil drainage classes in Denmark", Geoderma, Vol. 352, pp. 351-359, 2019.

[22] L. Estelles-Lopez, A. Ropodi, D. Pavlidis, J. Fotopoulou, C. Gkousari, A. Peyrodie, E. Panagou, G. J. Nychas, and F. Mohareb, “An automated ranking platform for machine learning regression models for meat spoilage prediction using multi-spectral imaging and metabolic profiling", Food Research International, Vol. 99, pp. 206-215, 2017.

[23] N. Shiri Harzevili and S. H. Alizadeh, "Mixture of latent multinomial naive Bayes classifier", Applied Soft Computing Journal, Vol. 69, pp. 516-527, 2018.

[24] D. Seka, B. S. Bonny, A. N. Yoboué, S. R. Sié, 
and B. A. Adopo-Gourène, "Identification of maize (Zea mays L.) progeny genotypes based on two probabilistic approaches: Logistic regression and naïve Bayes", Artificial Intelligence in Agriculture, Vol. 1, pp. 9-13, 2019.

[25] S. I. Sabilla, R. Sarno, and J. Siswantoro, "Estimating Gas Concentration using Artificial Neural Network for Electronic Nose", Procedia Computer Science, Vol. 124, pp. 181-188, 2017.

[26] G. Jiang and W. Wang, "Error estimation based on variance analysis of k-fold cross-validation", Pattern Recognition, Vol. 69, pp. 94-106, 2017.

[27] J. Xu, Y. Zhang, and D. Miao, "Three-way confusion matrix for classification: A measure driven view", Information Sciences, Vol. 507, pp. 772-794, 2020.

[28] Y. Potharaju, M. Kamsali, and C. R. Kesavari, "Classification of Ontological Violence Content Detection through Audio Features and Supervised Learning", International Journal of Intelligent Engineering and Systems, Vol. 12, No. 3, pp. 20-230, 2019.

[29] S. I. Sabilla, R. Sarno, and K. Triyana, "Optimizing Threshold Using Pearson Correlation for Selecting Features of Electronic Nose Signals", International Journal of Intelligent Engineering and Systems, Vol. 12, No. 6, pp. 81-90, 2019. 